\title{
Vertical and Lateral Separation
}

\author{
J. D. Proctor
}

Ir is generally assumed that the ideal distribution for the lateral or vertical distribution of aircraft on neighbouring tracks is as in Fig. I, where A and B are the centre lines of the routes or altitudes. Navaids and altimeters seem to be designed with this distribution as the ideal. Where air traffic control is imperfect and there is an appreciable risk that two aircraft without longitudinal separation will be at the same nominal altitude on the same route, then I suggest that neither the distribution of Fig. I nor that of Fig. 2 is ideal but that Fig. 3 represents the

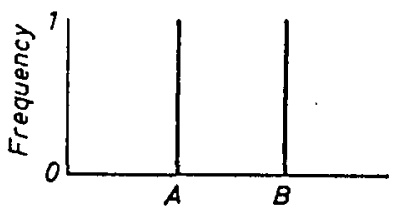

FIG. I

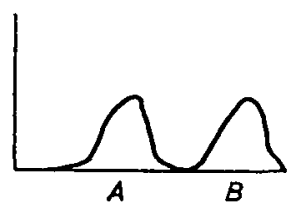

FIG. 2

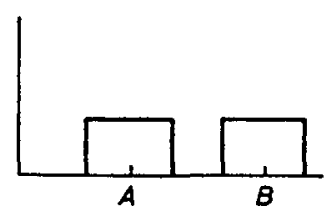

FIG. 3

ideal because it minimizes the risk of collision due to air traffic control failure. This hypothesis has implications for the design of navaids and altimeters and for the specification of vertical and lateral separation procedures.

\section{A Nocturnal}

\section{W. G. Webster}

INVESTIGATION into the design and accuracy of old-time instruments is always of interest. The Nocturnal probably used from the sixteenth century onwards for estimating the approximate local time at night is well known; with this primitive instrument the Pole Star was viewed through a central hole in a pair of wooden or brass discs. A pivoted arm turned to coincide with the 'Guards' of the Lesser Bear, the local time being deduced from an engraved scale. A seventeenthcentury improvement included an extra scale for determining the time by aligning the pointer with the Great Bear 'Pointers'.

The instrument could only have given a very approximate answer because its accuracy depended on its being held in the correct position, with the plane of the discs orthogonal to the line of sight to Polaris and, most important of all, with a small pointer on the periphery of the outer 'Calendar' scale (opposite 18 February) directed towards the observer's zenith. An error of $I^{\circ}$ in this pointer's direction would obviously have affected the answer by about 4 minutes. In addition the viewing hole seems to have been made in the centre of the discs and therefore with no allowance for the eccentricity of Polaris from the celestial pole (then about $3 \frac{1}{2}^{\circ}$ ). 
I have recently made a modern reproduction of the nocturnal and carried out trials to ascertain how accurately the time may be read. Fig. 2 shows the side towards the observer. I have purposely not added any extras (such as spirit level or mirrors) that were not then in use by mariners, but improvements which have been included are:

(i) The viewing hole offset from the true centre of the discs to allow for the present polar distance of Polaris (approx. $5^{\prime}$ ).

(ii) The pointer being double ended so that one end (D) can be directed at Dubhe if visible or the other end (B) at $\beta$ Cassiopeiae, should the former be obscured by cloud or below the horizon in low latitudes.

(iii) A short hinged flap which, when the nocturnal is held up in its correct position, can be set or allowed to hang. downwards but at the same time to turn with the outer fixed disc; from the upper hinged end of this flap hangs a short white plumb line. By keeping a painted white datum line on the flap coincident with the plumb line the observer can ensure that the disc is correctly orientated.

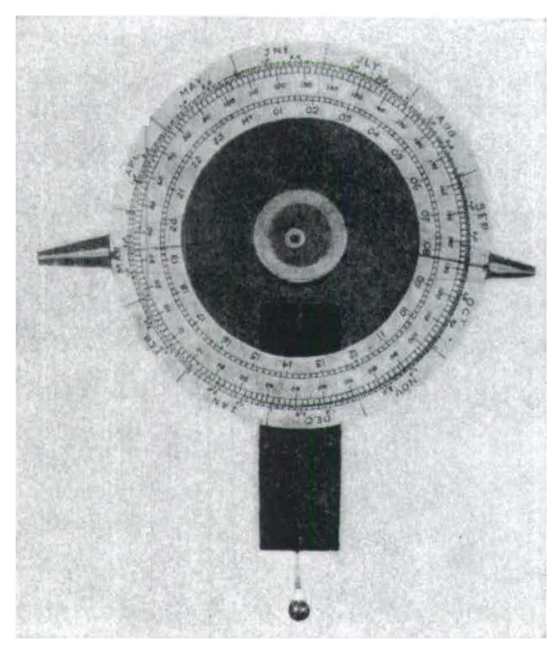

FIG. I

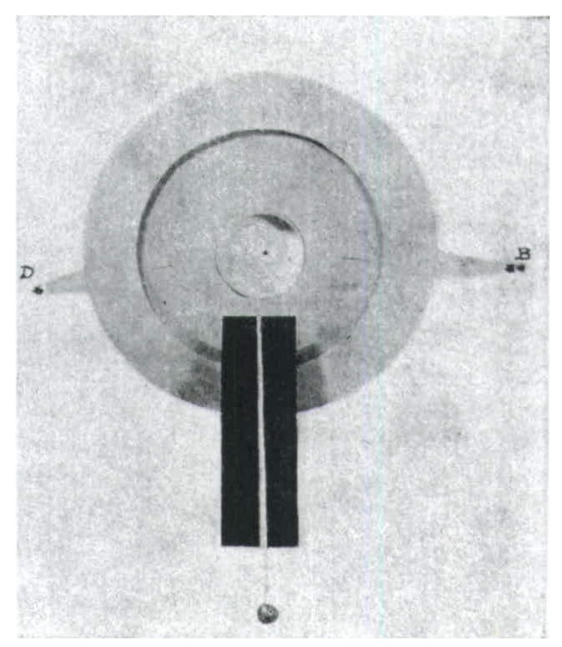

FIG. 2

(iv) Simplified scales: the outer calendar scale, drawn for the normal year, much the same as the original (a small allowance can be made for position in the leap year cycle); both a longitude scale and a time scale on the same (inner) disc, so that the observer can preset his longitude against the date and subsequently read off G.M.T. direct.

For easier identification and viewing, the discs and the pointer are made transparent except for the scaled portion. The viewing hole is made more visible by a ring of white or luminous paint, while at each end of the arm short white pointers are painted to assist contact with the required star.

In trials ashore 27 consecutive observations gave the following results :

$$
\begin{array}{ll}
\text { Maximum errors } & +7 \mathrm{~min} \text {, to }-6 \mathrm{~min} \text {. } \\
\text { Mean error } & +2 \mathrm{~min} .
\end{array}
$$


I estimate that the error of the original instrument in ships of that time probably varied from about $30 \mathrm{~min}$. in moderate seas down to about $10 \mathrm{~min}$. when absolutely calm. The error of single observations was likely to be higher than these.

The nocturnal obviously has absolutely no practical value today, ashore or afloat, except from an educational point of view. For such a purpose, I expect to produce some in the hope that some of our young people may be encouraged to look upwards to see some of the grandeur, orderly procession and stability of Creation and away from much of the man-made troubles that discourage them in their search for purpose in their lives.

\section{BIBLIOGRAPHY}

Cotter, C. H. (1967). A History of Nautical Astronomy.

Taylor, E. G. R. and Richey, M. W. (1962). The Geometrical Seaman. 